\title{
Editor's Note: Special issue on advanced P2P security and privacy
}

Published online: 7 May 2015

(C) Springer Science+Business Media New York 2015

The Peer-to-Peer Networking and Applications journal gratefully acknowledges the editorial work of the following scholars on this special issue "Advanced P2P Security and Privacy":

Dr. Chang Hoon Lee, SNUT, Korea

Dr. Shiuh-Jeng Wang, Central Police University, Taiwan

Dr. Liudong Xing, University of Massachusetts Dartmouth, USA

The seven papers in this section include:

- Securing network coding against pollution attacks in P2P converged ubiquitous networks, by Ming $\mathrm{He}$, Zhenghu Gong, Lin Chen, Hong Wang, Fan Dai, Zhihong Liu

- LSC ${ }^{2}$ : An extended link state protocol with centralized control, by Dan Zhao, Chunqing Wu, Xiaofeng $\mathrm{Hu}$, Hongjun Liu
- Merging sub-networks in VANETs by using the IEEE 802.11xx protocols, by Cándido Caballero-Gil, Pino Caballero-Gil, Jezabel Molina-Gil

- An efficient secure authentication scheme with user anonymity for roaming user in ubiquitous networks, by Soobok Shin, HongjinYeh, Kangseok Kim

- An approach to mitigate DoS attack based on routing misbehavior in wireless ad hoc networks, by Gunhee Lee, Wonil Kim, Kangseok Kim, Sangyoon Oh, Dong-kyoo Kim

- A study on memory dump analysis based on digital forensic tools, by JungtaekSeo, Seokjun Lee, Taeshik Shon

- Network processor architecture with flow-based dynamic bandwidth control for efficient QoS provisioning, by Jongsu Park, Yong-Surk Lee

- Weakness of lightweight block ciphers mCrypton and LED against biclique cryptanalysis, by KitaeJeong, HyungChul Kang, Changhoon Lee, Jaechul Sung, Seokhie Hong, Jong In Lim 